\title{
Effect of SOCS3 on apoptosis of human trophoblasts via adjustment of the JAK2/STAT3 signaling pathway in preterm birth
}

\author{
Yin Yin ${ }^{1}$, Lin $Q u^{1}$, Dicong Zhu ${ }^{2}$, Yang $\mathrm{Wu}^{1}$, Xin Zhou ${ }^{1}$ \\ ${ }^{1}$ Department of Obstetrics and Gynecology, The First Affiliated Hospital of Nanjing Medical University, Nanjing, China; ${ }^{2}$ State Key Laboratory of \\ Reproductive Medicine, Nanjing Medical University, Nanjing, China \\ Contributions: (I) Conception and design: Y Yin, X Zhou; (II) Administrative support: X Zhou; (III) Provision of study materials or patients: Y Yin, \\ L Qu; (IV) Collection and assembly of data: Y Yin, L Qu; (V) Data analysis and interpretation: All authors; (VI) Manuscript writing: All authors; (VII) \\ Final approval of manuscript: All authors. \\ Correspondence to: Xin Zhou. Department of Obstetrics and Gynecology, The First Affiliated Hospital of Nanjing Medical University, No. 368 North \\ Jiangdong Road, Nanjing 210036, China. Email: zhouxin101@sina.com.
}

Background: The expression of suppressor of cytokine signaling 3 (SOCS3) was induced by interleukin-6 (IL-6) in preterm placental tissues. However, its role in IL-6 induced apoptosis of trophoblast cells derived from preterm placental tissues remains to be elucidated.

Methods: Primary cytotrophoblasts from human preterm placental tissues were used to stably knock down and overexpress the level of SOCS3 by corresponding lentiviral vectors and the expression of SOCS3 was validated by quantitative reverse transcription polymerase chain reaction (qRT-PCR) and Western blot. The effect of SOCS3 overexpression or knockdown on the proliferation and apoptosis of IL-6 treated human cytotrophoblasts were determined by Cell Counting Kit-8 (CCK8) assay and Annexin-V/Propidium Iodide (PI) double-staining assay, respectively. Based on it, we detected the proteins associated with the Janus Tyrosine Kinase (JAK)/Signal Transducer and Activator of Transcription (STAT) pathway and apoptosis, such as JAK2, p-JAK2, STAT3, p-STAT3, B-cell lymphoma-2 (Bcl-2) and BCL2-associated X (Bax) by Western blot.

Results: IL-6-treatment resulted in significant apoptosis of human cytotrophoblasts. Overexpressing SOCS3 in the cytotrophoblasts reduced cell apoptosis, while the knockdown of SCOS3 had the opposite effects. Further analyses showed that SOCS3 overexpression inhibited JAK2 and STAT3 phosphorylation, which was induced by IL-6 stimulation.

Conclusions: SOCS3 plays a protective role in human preterm placental tissue-derived cytotrophoblasts from IL-6 induced apoptosis by feedback inhibition of JAK2/STAT3 signaling.

Keywords: Preterm birth; suppressor of cytokine signaling 3 (SOCS3); interleukin-6 (IL-6); apoptosis; JAK2/ STAT3 signaling

Submitted Jan 29, 2021. Accepted for publication May 12, 2021.

doi: $10.21037 / \mathrm{tp}-21-39$

View this article at: http://dx.doi.org/10.21037/tp-21-39

\section{Introduction}

Preterm birth, which refers to an actual gestation of between 20 and 37 weeks, is a major obstetric and global health problem and is the leading cause of child mortality in those under 5 years of age worldwide (1). Globally, the morbidity rate of preterm birth is $5-15 \%$, with approximately
15 million babies being born prematurely and more than 1 million deaths due to related complications every year (2). Also, preterm birth leads to adverse health outcomes for the mother and the baby and significant healthcare costs (3). According to the March of Dimes, a premature baby spends an average of 25.4 days in a specialty care nursery. The costs associated with premature birth increase the United States 
healthcare expenses by 26.2 billion dollars annually (4). However, preterm birth pathogenesis is poorly understood, and accumulating evidence suggests that it is a complex process with multiple attributable causes (5-7).

Preterm birth is classified into two groups: spontaneous and iatrogenic premature births, with $~ 75 \%$ occurring spontaneously. Iatrogenic preterm birth is caused by human intervention, while a solid body of evidence indicates that systemic inflammation or infection are causally linked to early spontaneous preterm birth (8-10). It is generally believed that infection induces uterine contractions, premature rupture of membranes, or cervical softening by promoting changes in prostaglandins, matrix metalloproteinases, or oxytocin receptors through cytokines secretion. Additionally, an important mechanism involved in the pathogenesis of premature birth is cytokine-mediated trophoblast cell apoptosis (11-13). Abrahams et al. have demonstrated that ligation of toll-like receptor 4 (TLR4) induces cytokine production, while TLR2 activation induces trophoblast cell death during certain intrauterine infections. They also showed that the apoptosis was dependent upon the inhibition of the $\mathrm{X}$-linked apoptosis protein (XIAP) and the activation of Caspase-8, -9, and -3 (14). More recently, numerous studies have also indicated that cytokines, such as tumor necrosis factor-alpha (TNF- $\alpha$ ), interferon-gamma (IFN- $\gamma$ ), and interleukin-1beta (IL-1 $\beta$ ) will cause physical and biochemical changes in trophoblast cells by inducing apoptosis and inflammatory reactions (15). Therefore, it is believed that excessive apoptosis of trophoblast cells plays a pivotal role in preterm birth pathogenesis; however, the detailed molecular mechanisms of apoptosis require further elucidation.

SOCS 3 is a member of the SOCS family, including SOCS1-7 and cytokine-inducible $\mathrm{SH} 2$ containing protein $(\mathrm{CISH})$, and negatively regulates cytokine signaling. The structural features of SOCS proteins include a conserved motif (termed the SOCS box of C terminal), a central Src homology-2 (SH2) area, and an N-terminal variable area (16-18). Roberts et al. reported that SOCS3 deficiency leads to midgestational fetal demise due to placental insufficiency (19). It is well accepted that SOCS3 is primarily induced by IL-6 family cytokines, such as IL-6, IL-11, leptin, and leukemia inhibitory factor (LIF), via the glycoprotein 130 (gp130)/JAK/STAT3 signaling pathway and inhibits the phosphorylation of JAK and STAT3 (20-22). The activation of JAK/STAT3 signaling is related to multiple biological functions, including immune regulation, cell proliferation, differentiation, hematopoiesis, and apoptosis (including SOCS3 gene transcription) (23). SOCS3 regulates the JAK/STAT3 signaling pathway by inhibiting inflammation and is also related to apoptosis. Some studies have shown that the knockdown of SOCS3 persistently activates STAT3 by upregulating the expression of growth regulatory proteins (Cyclin D1, c-Myc, c-Fos) or anti-apoptotic proteins [Bcl-2, myeloid cell leukemia 1 (Mcl-1), B-cell lymphoma extra-large (Bcl-XL)], thereby inducing hyperproliferation and preventing apoptosis $(24,25)$. Recent studies have demonstrated that hypermethylation of the $\mathrm{CpG}$ islands of the SOCS3 gene leads to SOCS3 silencing and promotes the growth and migration of human hepatocellular carcinoma, head and neck squamous cell carcinoma, and lung cancer $(26,27)$. As a negative cytokine signaling feedback inhibitor, SOCS3 is highly expressed in preterm placental trophoblast cells $(20,28)$. However, there are few studies on the mechanism underlying the apoptosis of SOCS3 in trophoblast cells.

Therefore, this study aimed to explore the role of SOCS3 in the apoptosis of placental trophoblast cells and its effects on the JAK2/STAT3 signaling pathway. Collectively, our data confirmed that IL-6 treatment of trophoblasts significantly promoted the expression of SOCS3 through the JAK2/STAT3 pathway. The expression of SOCS3 was required to inhibit IL-6-induced inflammation and trophoblast apoptosis. Our findings could strengthen the understanding of preterm birth pathogenesis mechanisms and provide theoretical guidance for subsequent studies.

We present the following article in accordance with the MDAR reporting checklist (available at http://dx.doi. org/10.21037/tp-21-39).

\section{Methods}

\section{Placenta collection}

Placentas were collected from cesarean sections of nonsmoking, healthy women with a pregnancy less than 37 weeks' gestation (hereafter called "preterm labor"). The placentas were obtained from the Jiangsu Women and Children Health Hospital. The study was conducted in accordance with the Declaration of Helsinki (as revised in 2013). The study was approved by the ethics committee of the First Affiliated Hospital of Nanjing Medical University (No. 2020-SR-434) and informed consent was taken from all the patients. 


\section{Cytotrophoblast purification and culture}

After placenta collection, the tissues were washed in phosphate buffered saline (PBS). Next, chorionic villi were gently scraped free from vessels and connective tissues, and were subsequently dissected into small fragments. The time for placental dissection was kept under 30 minutes to prevent tissue degradation. After dissection, the chorionic villi were washed twice with PBS and then digested by medium containing $0.1 \%$ Trypsin, $0.1 \mathrm{M}$ magnesium sulfate $\left(\mathrm{MgSO}_{4}\right), 0.1 \mathrm{M}$ calcium chloride $\left(\mathrm{CaCl}_{2}\right)$, $4 \%$ bovine serum albumin (BSA), and 50 Kunitz/mL Deoxyribonuclease I (DNase I) for 30 minutes at $37{ }^{\circ} \mathrm{C}$ without agitation. The supernatant-containing mononucleated villous cytotrophoblasts (VCTs) were collected after tissue sedimentation, filtered with a $40-\mu \mathrm{m}$ cell strainer, and incubated with fetal bovine serum (FBS) to stop trypsin activity. After purification by Percoll gradient centrifugation, the VCTs were resuspended and cultured in Dulbecco's Modified Eagle Medium (DMEM) supplemented with $10 \% \mathrm{FBS}, 2 \mathrm{mM}$ glutamine, $100 \mathrm{IU} / \mathrm{mL}$ penicillin, and $100 \mu \mathrm{g} / \mathrm{mL}$ streptomycin. After around 16 hours of culturing (overnight), the VCTs were carefully washed to eliminate non-adherent cells. The VCTs were characterized for each culture to ensure the purifications by microscopic visualization or collected and kept at $-80{ }^{\circ} \mathrm{C}$ for total ribonucleic acid (RNA) or protein extraction and fixed in cold methanol for further immunolabeling.

\section{Construction of SOCS3-overexpression or SOCS3- knockdown vectors}

Vector pCDH-MSCV-EF1-copGFP-T2A-Puro was used to construct the SOCS3 overexpressing plasmid (SOCS3OE). Briefly, coding sequences of human SOCS3 were amplified from human complementary DNA (cDNA) by polymerase chain reaction (PCR) using oligonucleotide to create restriction sites for EcoR I and BamH I of the SOCS3 sequence. The upstream (5'-AGCGCTAGCACCA TGGTCACCCACAGCAAGTTTCC-3') and downstream (5'-GGTGGTACCCAAAGCGGGGCATCGTACTG-3') primers were designed according to the National Center for Biotechnology Information (NCBI) and were synthesized by Sangon Biotech (Shanghai, China). PCR was performed using Pfu DNA polymerase (GENEWIZ, China). The PCR product and the pCDH-MSCV-EF1-copGFPT2A-Puro were digested with EcoR I and BamH I (NEB, Ipswich, MA). After ligation, competent DH5a E. coli cells were transformed using the plasmid, and the selected positive clones were amplified and verified by RuiBiotech (Beijing, China).

Vector pLKO.1-EGFP-puro was used to construct the SOCS3 knockdown plasmid (SOCS3-sh). SOCS3 short hairpin RNA (shRNA) and non-targeting shRNA control (sh-scramble) oligonucleotides were designed according to the Sigma website (https://www.sigmaaldrich.com/lifescience/functional-genomics-and-rnai/shrna/individualgenes.html) and were synthesized by Sangon Biotech (Shanghai). Oligonucleotides were annealed, and the pLKO.1-EGFP-puro were digested with EcoR I and Age I (NEB, Ipswich, MA). After ligation, competent DH5a $E$. coli cells were transformed using the plasmid and selected positive clones were amplified and verified by RuiBiotech (Beijing, China).

\section{Knockdown and overexpression of SOCS3 in buman cytotrophoblasts}

Lentivirus was produced by co-transfecting HEK293T cells with helper plasmids, including psPAX2 and pMD2G, and overexpressing (pCDH-MSCV-SOCS3-EF1-copGFPT2A-Puro) or knockdown (pLKO.1-shSOCS3-EGFPpuro) plasmids using polyethylenimine (PEI) reagent. Culture supernatants were collected after 48 hours, filtered using a $0.45-\mu \mathrm{m}$ filter, and the lentivirus collected from the supernatant was directly used to infect cells. To validate the efficiency of infection, quantitative reverse transcription polymerase chain reaction (qRT-PCR) and western blot analysis were used to examine the expression level of SOCS3 in human cytotrophoblasts.

\section{$R N A$ extraction and $q R T-P C R$}

Total RNA was extracted from human cytotrophoblasts using TRIzol reagent (Invitrogen, Carlsbad, CA, USA) according to the manufacturer's protocol. RNA was reversely transcribed into cDNA using a reverse transcription kit and then stored at $-80{ }^{\circ} \mathrm{C}$ until use. Also, qRT-PCR was performed using SYBR Green Mix (Transgen Biotech, Beijing, China). The PCR products were subjected to melting curve analysis to confirm the amplification. Messenger RNA (mRNA) with an amount of $20 \mathrm{ng}$ was normalized to $\beta$-actin and evaluated using the $2^{-\Delta \Delta C t}$ method. All experiments were performed in triplicate. The primer sequences were used as follows:

SOCS3-Forward: 5'-CATCTCTGTCGGAAGACCGT 


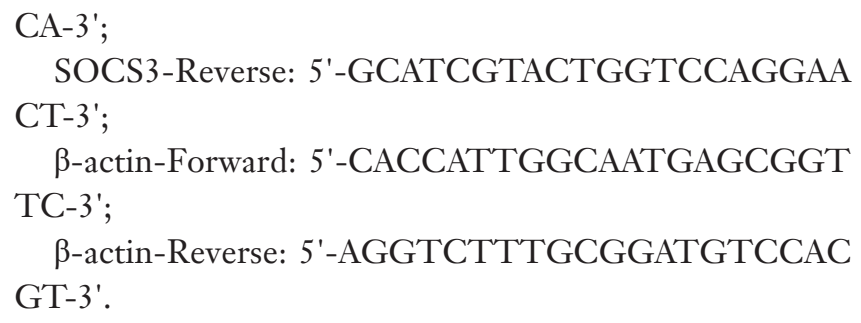

$\beta$-actin-Reverse: 5'-AGGTCTTTGCGGATGTCCAC GT-3'.

\section{Western blot analysis}

Whole cells were lysed in radioimmunoprecipitation assay (RIPA) lysis buffer containing protease inhibitor and phosphatase inhibitor for 30 minutes on ice, centrifuged at 12,000 rpm, and harvested proteins. Protein concentrations were measured using a bicinchoninic acid (BCA) protein assay kit (Pierce; Thermo Fisher Scientific, Inc.). Equal amounts of protein $(30 \mu \mathrm{g})$ were separated by $10 \%$ sodium dodecyl sulfate polyacrylamide gel electrophoresis (SDSPAGE) and transferred onto polyvinylidene difluoride membranes (PVDF; Millipore, Burlington, MA, USA). The membranes were then blocked in 5\% non-fat dry milk for 1 hour at room temperature in Tris-Buffered Saline and Tween 20 (TBST, 0.1\% Tween 20). The membranes were subsequently incubated with primary antibodies against SOCS3 (Cell Signaling Technology, Cat No. 52113S, 1:1,000), Bcl-2 (Proteintech, Cat No. 12789-1-AP, 1:1,000), Bax (Proteintech, Cat No. 50599-2-Ig, 1:1,000), p-JAK2 (Tyr1007/1008) (Cell Signaling Technology, Cat No. 3776S, 1:500), JAK2 (Proteintech, Cat No. 17670-1-AP, 1:1,000), p-STAT3 (Ser727) (Cell Signaling Technology, Cat No. 9136S, 1:1,000), STAT3 (Cell Signaling Technology, Cat No. 9139S, 1:1,000), and $\beta$-Actin (Proteintech, Cat No. $66009-1-\mathrm{Ig}, 1: 2,000)$ overnight at $4{ }^{\circ} \mathrm{C}$. The membranes were then washed with TBST three times and incubated with secondary horseradish peroxidase (HRP)-conjugated goat anti-Rabbit immunoglobulin G $(\mathrm{IgG})(\mathrm{H}+\mathrm{L})$ (Cell Signaling Technology, Cat No. 7074S, 1:2,000) or secondary HRP-conjugated horse anti-Mouse IgG $(\mathrm{H}+$ L) (Cell Signaling Technology, Cat No. 7076S, 1:2,000) at room temperature for 1 hour. All membranes were detected using an enhanced chemiluminescence kit (Millipore, Burlington, MA, USA), and the images were obtained using Image Lab software (Bio-Rad, USA). Blots were semiquantified using ImageJ software (National Institutes of Health).

\section{Flow cytometry}

The rate of cell apoptosis was detected by flow cytometry. After $24 \mathrm{~h}$ treatment with IL-6, lentivirus infected human cytotrophoblasts were washed with PBS solution and then dissociated with trypsin without edetate disodium (EDTA), and FBS was used to stop trypsin activity. After centrifugation at 2,000 rpm for 5 minutes, the supernatant was removed, and the cells were resuspended with $1 \times$ binding buffer. After the addition of $5 \mu \mathrm{L}$ Annexin V-APC and $5 \mu \mathrm{L}$ PI, the cells were incubated at $4{ }^{\circ} \mathrm{C}$ in the dark for 20 minutes, followed by flow cytometry. A total of 10,000 cells were counted, and the rate of cell apoptosis was calculated accordingly. Simultaneously, cells that were not incubated with Annexin V-APC and PI served as the negative control. The data was analyzed using FlowJo software (version 7.6, FlowJo LLC, Ashland, OR).

\section{Statistical analysis}

Prism software (version 8.0, GraphPad Software, San Diego, CA) was used for statistical analysis. One- or twoway ANOVAs were performed to determine significance. Statistical differences were indicated as ${ }^{*} \mathrm{P}<0.05$, ${ }^{*} \mathrm{P}<0.01$, ${ }^{* * *} \mathrm{P}<0.001$, and ${ }^{* * *} \mathrm{P}<0.0001$. All quantitative data were presented as the mean \pm the standard error of the mean (SEM) from three independent experiments.

\section{Results}

\section{Overexpression or knockdown of SOCS3 in buman cytotrophoblasts}

To investigate the role of SOCS3 in human placental cells, we first performed lentivirus-mediated gene delivery in primary human cytotrophoblasts to overexpress or knockdown the SOCS3 gene with pCDH-MSCV-SOCS3EF1-copGFP-T2A-Puro or pLKO.1-shSOCS3-EGFPpuro, respectively. After 24 hours of infection, enhanced green fluorescent protein (EGFP) expression was detected by fluorescent microscopy, and the transfection efficiency was approximately $90 \%$ (Figure 1A). At 48 hours after transfection, both qRT-PCR and Western blot assay showed that, compared with the EGFP control group, SOCS3 expression was significantly up-regulated or down-regulated in the pCDH-MSCV-SOCS3-EF1-copGFP-T2A-Puro 
A
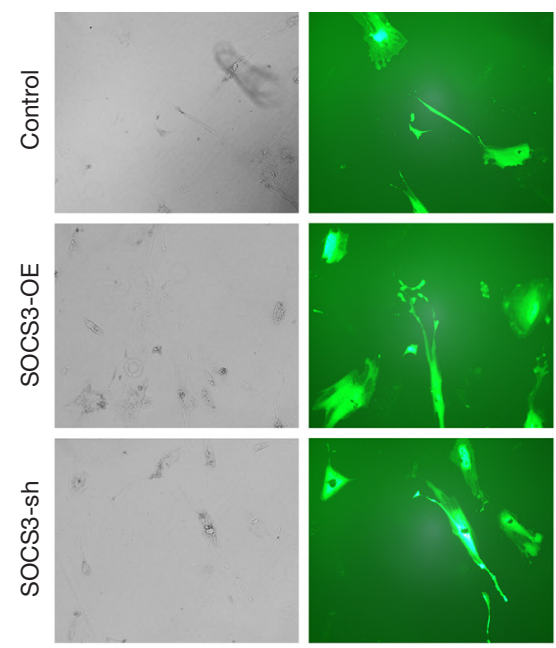

B

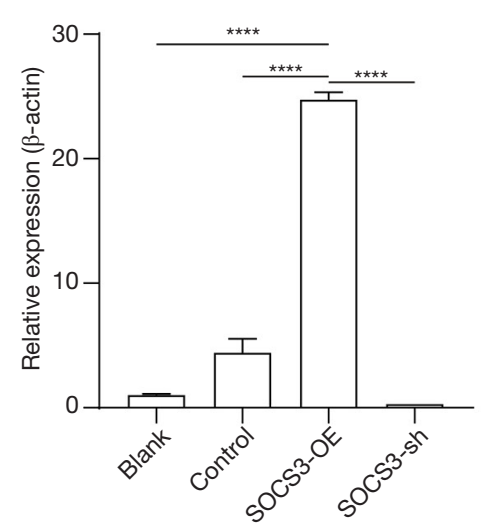

C

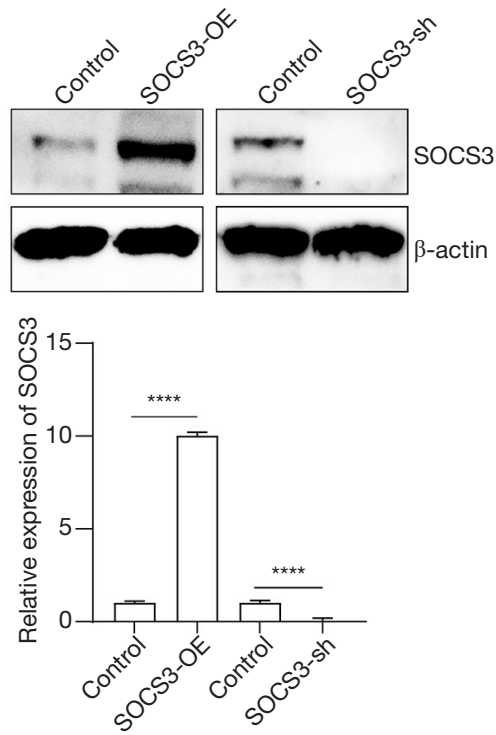

Figure 1 Successful overexpression or knockdown of SOCS3 in human cytotrophoblasts. (A) Representative photomicrographs demonstrating successful infection of human cytotrophoblasts with an empty vector (control), the PCDH-SCOS3 expression vector (SOCS3-OE), and PLKO-shSCOS3 knockdown vector (SOCS3-sh). Magnification, $\times 200$. (B) The SOCS3 mRNA level in the blank (nonlentivirus infection), control, SOCS3-OE, and SOCS3-sh was measured by qRT-PCR, and actin was used as control ( $\mathrm{n}=3$ per group). Oneway ANOVA, mean $\pm \mathrm{SEM},{ }^{* * * *} \mathrm{P}<0.0001$. (C) Representative blot demonstrating SOCS3 expression in human cytotrophoblasts following infection. $\beta$-actin was used as the loading control. (D) Blots were semi-quantified using ImageJ software. Data are expressed as the mean \pm SEM of the mean of three independent experiments. ${ }^{* * *} \mathrm{P}<0.0001$.

(SOCS3-OE) or pLKO.1-shSOCS3-EGFP-puro (SOCS3sh) groups, respectively (Figure 1B,C,D).

\section{The role of SOCS 3 in the survival of human cytotrophoblasts upon IL-6 exposure}

To determine the role of SOCS3 in the proliferation of human cytotrophoblasts upon IL-6 exposure, we treated normal SOCS3-OE and SOCS3-sh cells with $100 \mathrm{ng} / \mathrm{mL}$ IL-6 for 24 hours. IL-6 exposure significantly inhibited normal human cytotrophoblasts' survival, and the knockdown of SOCS3 genes (SOCS3-sh) showed additional inhibitory effects (Figure 2A). However, cells in the SOCS3-OE group exhibited markedly superior survival (Figure 2A), which suggested the expression of SOCS3 might play a protective role in human cytotrophoblasts upon IL-6 exposure. Furthermore, we performed a cell counting kit-8 (CCK-8) assay to evaluate human cytotrophoblasts' viability from the various treatment groups in vitro. As shown in Figure 2B, the viability of cells exposed to IL-6 was significantly decreased compared with the normal group $(\mathrm{P}<0.05)$, and the deletion of SOCS3 (SOCS3-sh) could promote this consequence.
Meanwhile, SOCS3 overexpression (SOCS3-OE) promoted the proliferation of human cytotrophoblasts, even in the presence of IL-6 (Figure 2B). These results suggested that enhanced cellular SOCS3 expression could reverse IL-6induced growth inhibition in human cytotrophoblasts.

\section{SOCS3 overexpression protected human cytotrophoblasts from IL-6-induced apoptosis}

To determine the role of SOCS3 in IL-6-induced apoptosis of human cytotrophoblasts, we exposed both SOCS3OE and SOCS3-sh cells to $100 \mathrm{ng} / \mathrm{mL}$ IL-6 for 24 hours. The fraction of apoptotic cells in the SOCS3-OE group was significantly reduced compared to the control group (Figure 3). Also, IL-6-induced Bax up-regulation and $\mathrm{Bcl} 2$ down-regulation were reversed in SOCS3-OE cells (Figure 4). These results indicated that overexpression of SOCS3 could reverse IL-6-induced apoptosis in human cytotrophoblasts.

On the other hand, compared with the control group, the proportion of apoptosis in the SOCS3-sh group increased slightly (Figure 3), and IL-6-induced Bax up-regulation and 

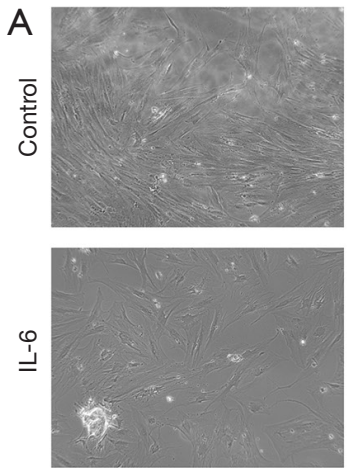
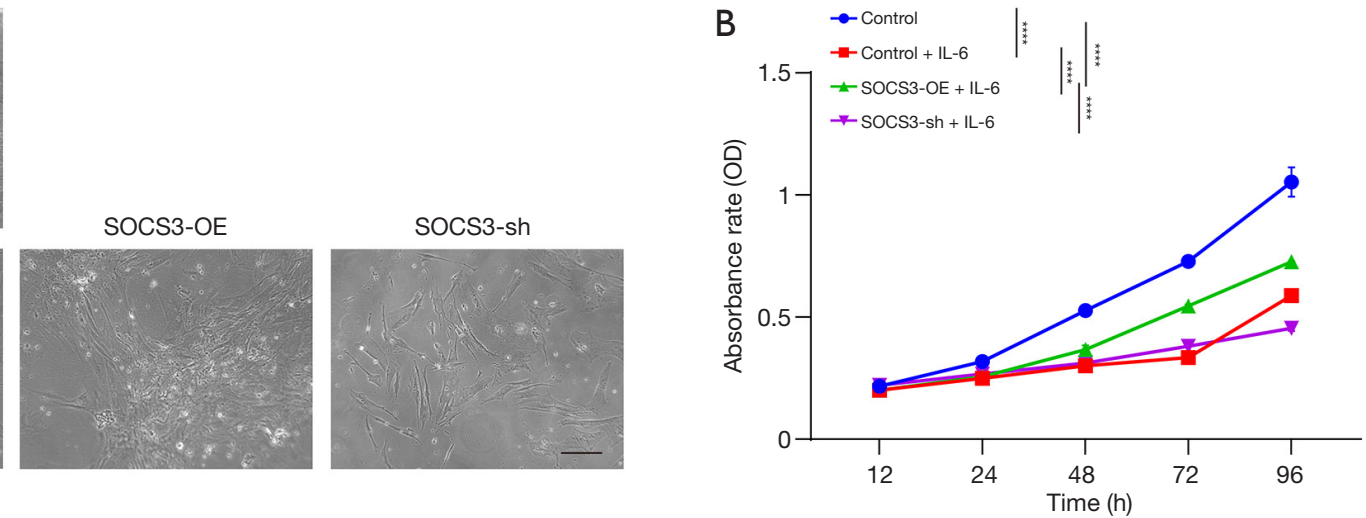

Figure 2 The role of SOCS3 in the survival of human cytotrophoblasts upon IL-6 exposure. (A) Representative photomicrographs of human cytotrophoblasts cultured in IL-6 stimuli. Bar $=20 \mu \mathrm{m}$. (B) Cell Counting Kit- 8 assay of human cytotrophoblasts following various treatments. Data are expressed as the mean \pm SEM of the mean of six independent measurements. ${ }^{* * * *} \mathrm{P}<0.0001$.

A
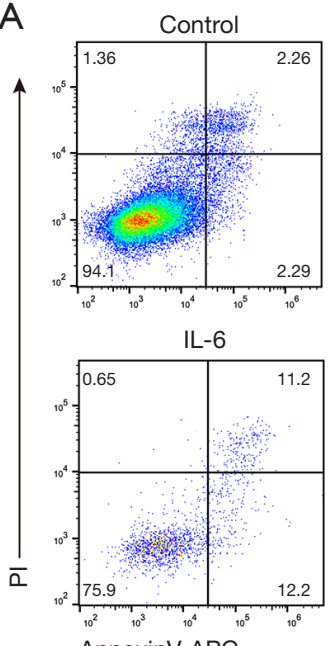

AnnexinV-APC

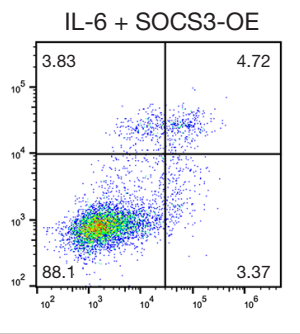

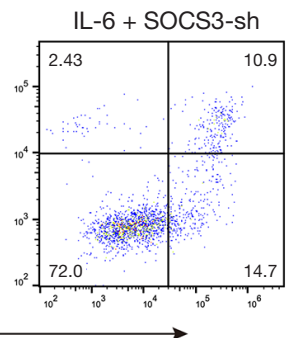

B

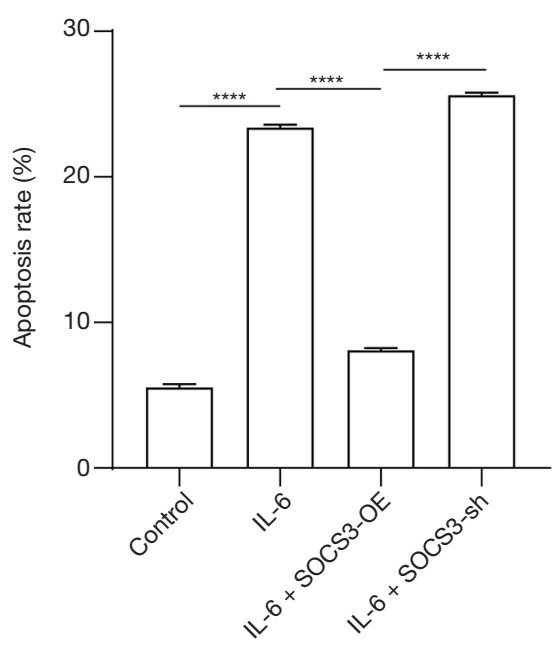

Figure 3 SOCS3 overexpression protected human cytotrophoblasts from IL-6-induced apoptosis. (A) The apoptosis rates of human cytotrophoblasts in the blank, IL-6 exposure, SCOS3-OE + IL-6, and SCOS3-sh + IL-6 groups were detected by flow cytometry. (B) Apoptosis cells of AnnexinV-APC/PI double staining. The data are presented as means \pm SEM of triplicates from three independent tests. **** $\mathrm{P}<0.0001$.

Bcl2 down-regulation were also promoted, although no statistical significance was observed. Therefore, SOCS3 knockdown had no significant additional effect on IL6 -induced apoptosis of human cytotrophoblasts. These results suggested that the feedback up-regulation of SOCS3 to a certain level might be essential to protect human cytotrophoblasts from IL-6-induced apoptosis.

\section{SOCS3/FAK/STAT signaling in the survival of buman cytotrophoblasts upon IL-6 stimuli}

To comprehensively understand the mechanism of SOCS3 in regulating the apoptosis of human cytotrophoblasts during IL-6 treatment, we further examined the JAK2/ STAT3 pathway. Western blot analysis showed that 
A

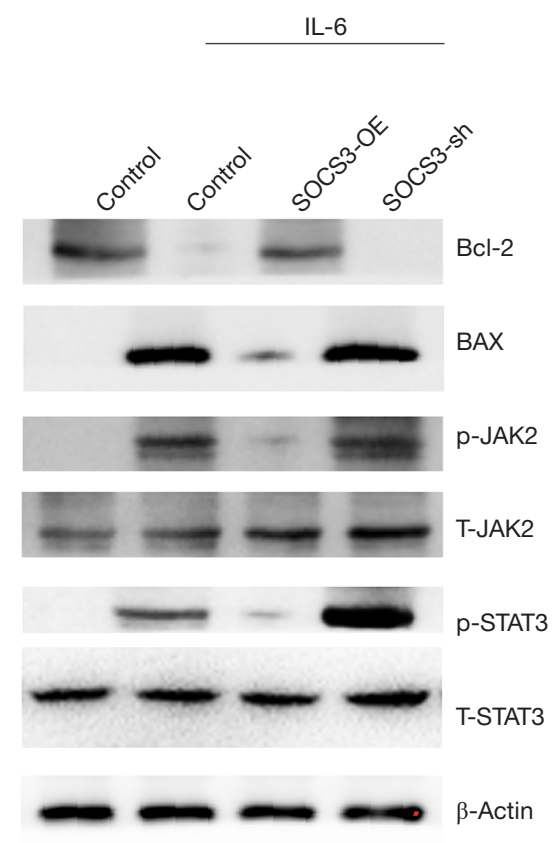

B

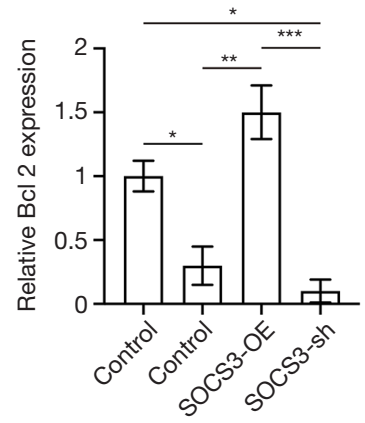

IL-6

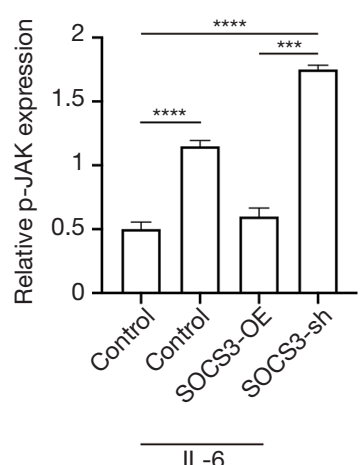

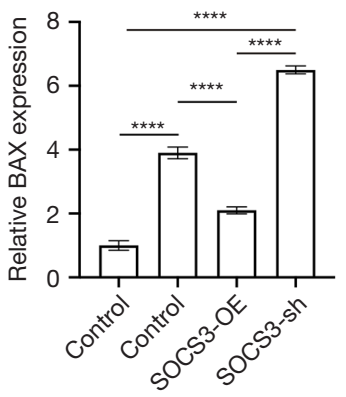

IL-6

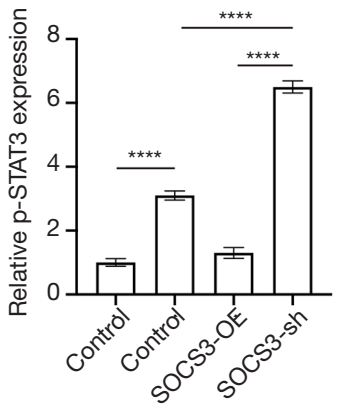

IL-6

Figure 4 SOCS3/JAK/STAT signaling in the survival of human cytotrophoblasts upon IL-6 stimuli. (A) The protein expression levels of apoptotic and JAK2-STAT3 genes. The data are presented from at least three independent tests. (B) The levels of Bcl-2, BAX, p-JAK2, and p-STAT3 were measured by ImageJ software and determined by taking the ratio of the target protein band intensity against that of $\beta$-actin. Data are expressed as the mean \pm SEM of the mean of three independent measurements. ${ }^{*} \mathrm{P}<0.05 ;{ }^{* *} \mathrm{P}<0.01 ;{ }^{* * *} \mathrm{P}<0.001 ;{ }^{* * * *} \mathrm{P}<0.0001$.

the phosphorylation of JAK2 and STAT3 in human cytotrophoblasts was significantly inhibited by SOCS3 overexpression during IL-6 treatment (Figure 4). On the other hand, the knockdown of SOCS3 marginally promoted these phosphorylated modifications. These results indicated that the JAK/STAT signaling pathway was involved in IL6 -induced apoptosis of human cytotrophoblasts and that negative feedback regulation by SOCS3 expression was required to promote the survival of human cytotrophoblasts.

\section{Discussion}

SOCS3 is a negative regulator of the JAK/STAT signaling transduction pathway. In this study, our results showed that IL-6 exposure induced apoptosis of human cytotrophoblasts. However, apoptosis tended to be reduced after SOCS3 overexpression and was significantly increased after SOCS3 knockdown. Further analyses showed that the overexpression of SOCS3 decreased the levels of phosphorylated JAK and STAT3, which were up-regulated by IL-6 stimulation.

IL-6 is a crucial modulator of mammalian responses to tissue damage caused by infection and inflammation, which are among the major causes of human preterm birth. Consistent with this, our previous study showed that the expression of IL- 6 was notably increased in preterm placental tissues (20). As SOCS3 played a negative feedback role in IL-6-induced activation of JAK/STAT3 signaling $(20,29)$, the overexpression of SOCS3 in preterm placental tissue-derived cytotrophoblasts also inhibited IL-6-induced p-STAT3, while SOCS3-knockdown had the opposite effect.

Given the high expression of SOCS3 and low expression of p-STAT3 in preterm placental tissues (20), it remains to be determined whether cytotrophoblast apoptosis is inhibited in vivo. Furthermore, it has been reported that SOCS3 can regulate the quantity and type of STAT signal 
generated from the IL-6 receptor (IL-6R), and the absence of SOCS3 significantly induces the phosphorylation of both STAT3 and STAT1 $(29,30)$. Thus, the phosphorylation status and exact roles of other STAT proteins in preterm placental tissues and cultured cytotrophoblasts remains to be determined.

On the other hand, both the fetal and the maternal immune systems were actively involved in maintaining normal pregnancy (31-33). It has been reported that IL-6 stimulation promotes STAT3 phosphorylation in both fetal and adult monocytes (34). Although fetal and adult monocytes express similar levels of SOCS3, the expression of IL-6R in adult monocytes was significantly lower than that in fetal monocytes, which resulted in markedly higher STAT3 phosphorylation in fetal monocytes exposed to IL-6. Illustration of the mechanism underlying IL-6/ STAT3/SOCS3 signaling regulation in both fetal and adult monocytes would provide new insights into inflammationassociated preterm birth.

Infection during pregnancy leads to increased expression of IL-6 and ultimately to a high expression level of SOCS3, which negatively regulates the JAK/STAT3 signaling pathway. During minor infections, SCOS3 can protect trophoblast cells and prevent premature delivery. However, during severe infection, the negative regulatory effect of SOCS3 is insufficient, and trophoblast cell apoptosis leads to premature birth. The expression of SOCS3 in patients with recurrent abortion is decreased (35); however, the specific mechanism remains unclear. It may be related to DNA methylation and other appearance modifications. Clarifying the mechanism of abnormally low SOCS3 expression may serve as a molecular marker for predicting recurrent abortion.

\section{Conclusions}

In summary, IL-6 induced apoptosis of human cytotrophoblasts, while the overexpression of SOCS3 exhibited protective effects. Further analyses showed that the overexpression of SOCS3 decreased the levels of phosphorylated JAK2 and STAT3, which were up-regulated by IL-6 stimulation. Our results suggest that SOCS3 is a potential therapeutic target for preterm births.

\section{Acknowledgments}

The authors would like to acknowledge Kassem A. and Chapnick J. for English language editing.
Funding: This work received financial support from the Jiangsu Maternal and Child Health Research Project (No. F201658).

\section{Footnote}

Reporting Checklist: The authors have completed the MDAR reporting checklist. Available at http://dx.doi.org/10.21037/ tp-21-39

Data Sharing Statement: Available at http://dx.doi. org/10.21037/tp-21-39

Peer Review File: Available at http://dx.doi.org/10.21037/tp21-39

Conflicts of Interest: All authors have completed the ICMJE uniform disclosure form (available at http://dx.doi. org/10.21037/tp-21-39). The authors have no conflicts of interest to declare.

Ethical Statement: The authors are accountable for all aspects of the work in ensuring that questions related to the accuracy or integrity of any part of the work are appropriately investigated and resolved. The study was conducted in accordance with the Declaration of Helsinki (as revised in 2013). The study was approved by the ethics committee of the First Affiliated Hospital of Nanjing Medical University (No. 2020-SR-434) and informed consent was taken from all the patients.

Open Access Statement: This is an Open Access article distributed in accordance with the Creative Commons Attribution-NonCommercial-NoDerivs 4.0 International License (CC BY-NC-ND 4.0), which permits the noncommercial replication and distribution of the article with the strict proviso that no changes or edits are made and the original work is properly cited (including links to both the formal publication through the relevant DOI and the license). See: https://creativecommons.org/licenses/by-nc-nd/4.0/.

\section{References}

1. Howson CP, Kinney MV, McDougall L, et al. Born too soon: preterm birth matters. Reprod Health 2013;10 Suppl 1:S1.

2. Lamont RF. Advances in the Prevention of InfectionRelated Preterm Birth. Front Immunol 2015;6:566. 
3. Wen SW, Guo Y, Rodger M, et al. Folic Acid Supplementation in Pregnancy and the Risk of PreEclampsia-A Cohort Study. PLoS One 2016;11:e0149818.

4. Evereklian M, Posmontier B. The Impact of Kangaroo Care on Premature Infant Weight Gain. J Pediatr Nurs 2017;34:e10-e16.

5. Armaly Z, Jadaon JE, Jabbour A, et al. Preeclampsia: Novel Mechanisms and Potential Therapeutic Approaches. Front Physiol 2018;9:973.

6. Burton GJ, Redman CW, Roberts JM, et al. Preeclampsia: pathophysiology and clinical implications. BMJ 2019;366:12381.

7. Tomimatsu T, Mimura K, Matsuzaki S, et al. Preeclampsia: Maternal Systemic Vascular Disorder Caused by Generalized Endothelial Dysfunction Due to Placental Antiangiogenic Factors. Int J Mol Sci 2019;20:4246.

8. Seo K, McGregor JA, French JI. Preterm birth is associated with increased risk of maternal and neonatal infection. Obstet Gynecol 1992;79:75-80.

9. Shim SS, Romero R, Hong JS, et al. Clinical significance of intra-amniotic inflammation in patients with preterm premature rupture of membranes. Am J Obstet Gynecol 2004;191:1339-45.

10. Nath CA, Ananth CV, Smulian JC, et al. Histologic evidence of inflammation and risk of placental abruption. Am J Obstet Gynecol 2007;197:319.e1-6.

11. Straszewski-Chavez SL, Abrahams VM, Mor G. The role of apoptosis in the regulation of trophoblast survival and differentiation during pregnancy. Endocr Rev 2005;26:877-97.

12. Nourollahpour Shiadeh M, Behboodi Moghadam Z, Adam I, et al. Human infectious diseases and risk of preeclampsia: an updated review of the literature. Infection 2017;45:589-600.

13. Kumar D, Fung W, Moore RM, et al. Proinflammatory cytokines found in amniotic fluid induce collagen remodeling, apoptosis, and biophysical weakening of cultured human fetal membranes. Biol Reprod 2006;74:29-34.

14. Abrahams VM, Bole-Aldo P, Kim YM, et al. Divergent trophoblast responses to bacterial products mediated by TLRs. J Immunol 2004;173:4286-96.

15. Caulfield MP, Robbins J, Brown DA. Neurotransmitters inhibit the omega-conotoxin-sensitive component of $\mathrm{Ca}$ current in neuroblastoma $\mathrm{x}$ glioma hybrid (NG 108-15) cells, not the nifedipine-sensitive component. Pflugers Arch 1992;420:486-92.

16. Carow B, Rottenberg ME. SOCS3, a Major Regulator of
Infection and Inflammation. Front Immunol 2014;5:58.

17. Klepsch O, Namer LS, Kohler N, et al. Intragenic regulation of SOCS3 isoforms. Cell Commun Signal 2019;17:70.

18. Page BD, Ball DP, Gunning PT. Signal transducer and activator of transcription 3 inhibitors: a patent review. Expert Opin Ther Pat 2011;21:65-83.

19. Roberts AW, Robb L, Rakar S, et al. Placental defects and embryonic lethality in mice lacking suppressor of cytokine signaling 3. Proc Natl Acad Sci U S A 2001;98:9324-9.

20. Zhou X, Jiang Z, Zou Y, et al. Role of SOCS3 in the Jak/ stat3 pathway in the human placenta: different mechanisms for preterm and term labor. Acta Obstet Gynecol Scand 2015;94:1112-7.

21. San Martin S, Fitzgerald JS, Weber M, et al. Stat3 and Socs 3 expression patterns during murine placenta development. Eur J Histochem 2013;57:e19.

22. Isobe A, Takeda T, Sakata M, et al. STAT3-mediated constitutive expression of SOCS3 in an undifferentiated rat trophoblast-like cell line. Placenta 2006;27:912-8.

23. Fletcher S, Drewry JA, Shahani VM, et al. Molecular disruption of oncogenic signal transducer and activator of transcription 3 (STAT3) protein. Biochem Cell Biol 2009;87:825-33.

24. Lu Y, Fukuyama S, Yoshida R, et al. Loss of SOCS3 gene expression converts STAT3 function from anti-apoptotic to pro-apoptotic. J Biol Chem 2006;281:36683-90.

25. Ogata H, Kobayashi T, Chinen T, et al. Deletion of the SOCS3 gene in liver parenchymal cells promotes hepatitis-induced hepatocarcinogenesis. Gastroenterology 2006;131:179-93.

26. Sutherland KD, Lindeman GJ, Visvader JE. Knocking off SOCS genes in the mammary gland. Cell Cycle 2007;6:799-803.

27. Weber A, Hengge UR, Bardenheuer W, et al. SOCS-3 is frequently methylated in head and neck squamous cell carcinoma and its precursor lesions and causes growth inhibition. Oncogene 2005;24:6699-708.

28. Takahashi Y, Carpino N, Cross JC, et al. SOCS3: an essential regulator of LIF receptor signaling in trophoblast giant cell differentiation. EMBO J 2003;22:372-84.

29. Croker BA, Krebs DL, Zhang JG, et al. SOCS3 negatively regulates IL-6 signaling in vivo. Nat Immunol 2003;4:540-5.

30. Murray PJ. The JAK-STAT signaling pathway: input and output integration. J Immunol 2007;178:2623-9.

31. Erlebacher A. Immunology of the maternal-fetal interface. Annu Rev Immunol 2013;31:387-411. 
32. Burt TD. Fetal regulatory $T$ cells and peripheral immune tolerance in utero: implications for development and disease. Am J Reprod Immunol 2013;69:346-58.

33. Miller D, Gershater M, Slutsky R, et al. Maternal and fetal $\mathrm{T}$ cells in term pregnancy and preterm labor. Cell Mol Immunol 2020;17:693-704.

Cite this article as: Yin Y, Qu L, Zhu D, Wu Y, Zhou X. Effect of SOCS3 on apoptosis of human trophoblasts via adjustment of the JAK2/STAT3 signaling pathway in preterm birth. Transl Pediatr 2021;10(6):1637-1646. doi: 10.21037/tp-21-39
34. Krow-Lucal ER, Kim CC, Burt TD, et al. Distinct functional programming of human fetal and adult monocytes. Blood 2014;123:1897-904.

35. Ding Y, Fu L. Apoptosis Related Gene Expression Profile of Trophoblasts Derived from Recurrent Spontaneous Abortion. Chin J Cell Biol 2009;31:250-6. 